\title{
Altered intestinal microbiota and blood T cell phenotype are shared by patients with Crohn's disease and their unaffected siblings
}

\author{
Charlotte R Hedin, ${ }^{1,2}$ Neil E McCarthy, ${ }^{2}$ Petra Louis, ${ }^{3}$ Freda M Farquharson, ${ }^{3}$ \\ Sara McCartney, ${ }^{4}$ Kirstin Taylor, ${ }^{5}$ Natalie J Prescott, ${ }^{5}$ Trevor Murrells, ${ }^{6}$ \\ Andrew J Stagg, ${ }^{7}$ Kevin Whelan, ${ }^{1}$ James 0 Lindsay ${ }^{2,8}$
}

\begin{abstract}
- Additional material is published online only. To view please visit the journal online (http://dx.doi.org/10.1136/ gutjnl-2013-306226).

${ }^{1}$ Diabetes and Nutritional Sciences Division, School of Medicine, King's College London, London, UK ${ }^{2}$ Centre for Digestive Diseases, Blizard Institute, Queen Mary University of London, London, UK ${ }^{3}$ Microbiology Group, Gut Health Theme, Rowett Institute of Nutrition and Health, University of Aberdeen, Bucksburn, Aberdeen, UK ${ }^{4}$ Department for Gastroenterology and Clinical Nutrition, University College Hospitals NHS Foundation

Trust, London, UK

${ }^{5}$ Department of Medical and Molecular Genetics, King's College London, London, UK ${ }^{6}$ National Nursing Research Unit, Florence Nightingale School of Nursing and Midwifery, King's College London, London, UK

${ }^{7}$ Centre for Immunology and Infectious Disease, Blizard Institute, Queen Mary University of London, London, UK ${ }^{8}$ Gastroenterology Division, Barts Health NHS Trust, London, UK
\end{abstract}

\section{Correspondence to} Dr James Lindsay, Barts Health NHS Trust, Endoscopy Unit, The Royal London Hospital, Whitechapel, London E1 1BB, UK

James.Lindsay@bartshealth. nhs.uk

Received 9 October 2013 Revised 13 November 2013 Accepted 6 December 2013 Published Online First 7 January 2014

\section{CrossMark}

To cite: Hedin CR

McCarthy NE, Louis $P$, et al. Gut 2014;63:1578-1586.

\section{ABSTRACT}

Objective Crohn's disease (CD) is associated with intestinal dysbiosis, altered blood T cell populations, elevated faecal calprotectin (FC) and increased intestinal permeability (IP). CD-associated features present in siblings (increased risk of CD) but not in healthy controls, provide insight into early CD pathogenesis. We aimed to (1) Delineate the genetic, immune and microbiological profile of patients with $C D$, their siblings and controls and (2) Determine which factors discriminate between groups.

Design Faecal microbiology was analysed by quantitative PCR targeting $16 \mathrm{~S}$ ribosomal RNA, FC by ELISA, blood T cell phenotype by flow cytometry and IP by differential lactulose-rhamnose absorption in 22 patients with inactive CD, 21 of their healthy siblings and 25 controls. Subject's genotype relative risk was determined by Illumina Immuno BeadChip.

Results Strikingly, siblings shared aspects of intestinal dysbiosis with patients with CD (lower concentrations of Faecalibacterium prausnitzii ( $p=0.048)$, Clostridia cluster IV ( $p=0.003)$ and Roseburia spp. $(p=0.09)$ compared with controls). As in $C D$, siblings demonstrated a predominance of memory T cells $(p=0.002)$ and elevated naïve CD4 T cell $\beta 7$ integrin expression $(p=0.01)$ compared with controls. FC was elevated $(>50 \mu \mathrm{g} / \mathrm{g})$ in $8 / 21$ (38\%) siblings compared with 2/25 (8\%) controls $(p=0.028)$; whereas IP did not differ between siblings and controls. Discriminant function analysis determined that combinations of these factors significantly discriminated between groups $\left(\chi^{2}=80.4, \mathrm{df}=20\right.$ $p<0.001)$. Siblings were separated from controls by immunological and microbiological variables.

Conclusions Healthy siblings of patients with $C D$ manifest immune and microbiological abnormalities associated with CD distinct from their genotype-related risk and provide an excellent model in which to investigate early CD pathogenesis.

\section{INTRODUCTION}

Crohn's disease (CD) causes intestinal inflammation, occurs predominantly in young adults and results in significant morbidity and reduced quality of life. The pathogenesis of CD is unknown, but is thought to depend on abnormal interactions between the intestinal immune system and microbiota in genetically susceptible hosts. This model is supported by the identification of $>130$ risk loci associated with $\mathrm{CD},{ }^{1}$

\section{Significance of this study}

What is already known about this subject?

- Crohn's disease is associated with intestinal dysbiosis, T cell abnormalities and elevated genetic risk.

- The siblings of patients with Crohn's disease are at increased risk of developing disease compared with the background population.

- A proportion of the siblings of patients with Crohn's disease will have elevated faecal calprotectin and intestinal permeability.

\section{What are the new findings?}

- The siblings of patients with Crohn's disease share some aspects of the intestinal dysbiosis with their affected relatives such as reduced Firmicutes including Faecalibacterium prauznitsii which has been shown to be relevant to clinical course of disease.

- The siblings of patients with Crohn's disease share abnormalities in T cell phenotype with their affected relatives.

- Siblings of patients with Crohn's disease can be discriminated from healthy controls by a combination of immunological and microbiological factors.

\section{How might it impact on clinical practice in} the foreseeable future?

- This is the first study to assess multiple aspects of the Crohn's disease phenotype in patients' unaffected siblings and healthy controls.

- It identifies novel microbiological and immunological factors relevant to early disease pathogenesis.

- It raises the possibility of predicting the risk of Crohn's disease onset using multidimensional phenotyping.

including genes controlling microbial sensing and immune function. Furthermore, patients with CD exhibit altered intestinal microbiota (dysbiosis) including reduced Firmicutes such as Clostridia cluster IV and Faecalibacterium prausnitzii, ${ }^{2}$ and increased Escherichia coli. ${ }^{3} \mathrm{CD}$ is characterised 
immunologically by predominant Th1/Th17 mucosal $\mathrm{T}$ cell responses, ${ }^{4}$ with alterations in peripheral $\mathrm{T}$ cell populations and their expression of intestinal homing receptors such as $\alpha 4 \beta 7$ integrin. ${ }^{5}$ Finally, intestinal inflammation in $\mathrm{CD}$ is associated with a raised faecal calprotectin $(\mathrm{FC})^{7}$ and altered intestinal permeability (IP). ${ }^{8}$ Individual factors such as elevated FC or lower mucosal $F$ prausnitzii can predict natural history of CD disease. ${ }^{29}$ However, the alterations noted may be a consequence of established disease. This study aims to delineate features of individuals at risk of CD relevant to disease pathogenesis.

First-degree relatives of patients with $\mathrm{CD}$ are at increased risk of developing IBD compared with background populations. ${ }^{10}$ Monozygotic twin disease discordance highlights the importance of non-genetic factors in disease pathogenesis. Genotype alone therefore accounts for a significant but limited proportion of $\mathrm{CD}$ risk and biomarkers of non-genetic influences must be sought. ${ }^{11} 12$ Studying siblings of patients with CD may provide insights into genetic and non-genetic factors relevant to $C D$ pathogenesis, that are more readily apparent in the absence of established disease.

Several authors have sought to describe the phenotype of the 'at-risk' relatives of patients with CD. FC is increased in up to $49 \%$ of first-degree relatives, ${ }^{13}$ and IP is reportedly increased in $\mathrm{CD}$ relatives, although data are inconsistent. ${ }^{8}{ }^{14-16}$ Fewer data describe the intestinal microbiota of healthy relatives of patients with $\mathrm{CD}$; one study reported specific relative-associated dysbiosis distinct from that associated with $\mathrm{CD},{ }^{17}$ while other data have suggested an association between dysbiosis in relatives with CD and CD-associated alleles. ${ }^{18}$ However, the microbial characteristics of relatives' dysbiosis are inconsistent between studies.

Interaction between gut microbiota and the mucosal immune system is a cornerstone of CD pathogenesis, yet few data exist that define the immune phenotype of those at risk of CD. ${ }^{19}$ Defects in oral tolerance induction have been reported in some first-degree relatives of patients with $\mathrm{CD}^{20}$ but there has been no detailed analysis of the associated $\mathrm{T}$ cell phenotype and function.

Limitations of previous studies include recruitment of unaffected parents of patients with $\mathrm{CD}$, and the determination of specific aspects of the at-risk phenotype in isolation. The inclusion of siblings is preferable to parents because relevant risk factors such as raised $\mathrm{FC}^{21}$ and altered gut microbiota, ${ }^{22}$ may vary with age. Furthermore, parents are usually beyond the peak age of $\mathrm{CD}$ incidence, reducing the power to detect biomarkers of IBD susceptibility. Finally, comparison of individuals separated by a generation may be confounded by changes in prevailing environmental conditions. These issues are circumvented in the current study by comparing phenotypes of patients and their unaffected siblings.

The current study was designed to test the hypothesis that unaffected siblings of patients with $\mathrm{CD}$ share features of a ' $\mathrm{CD}$ phenotype' that may afford insights into disease pathogenesis and provide markers of CD risk. This study is unique by defining multiple dimensions of the CD phenotype simultaneously, including microbial and immunological markers alongside FC, IP and genotype. This has allowed interrogation of the relative impact and interactions of these features.

\section{MATERIALS AND METHODS}

Patients with inactive CD (Crohn's Disease Activity Index $(\mathrm{CDAI})<150$ and $\mathrm{C}$ reactive protein $(\mathrm{CRP})<5 \mathrm{mg} / \mathrm{L}$ ) and their healthy siblings (both aged 16-35 years) were recruited from clinics at Barts and London, and University College Hospitals NHS Trusts, UK between June 2008 and December 2011.
Healthy controls were recruited by circular email sent to staff and students at King's College London during the same period. Patients required a confirmed diagnosis of CD for $>3$ months and an available sibling. For exclusion criteria see online supplementary table S1. All participants provided written informed consent. Ethical approval was provided by Bromley Local Research Ethics Committee (reference 07/H0805/46).

At screening, demographics, medical and drug exposure history, physical examination, CRP, inclusion and exclusion criteria were assessed. Written instructions regarding avoidance of prebiotics/probiotics for 4 weeks, non-steroidal antiinflammatory drugs for 1 week and alcohol for $24 \mathrm{~h}$ before the study were provided. Blood samples were taken for routine haematology/biochemistry, $\mathrm{T}$ cell analysis and genotyping. Participants completed the 5-h urine collection for IP and underwent flexible rectoscopy without bowel cleansing. Biopsies from non-inflamed rectum were sent for routine histological analysis. Stool was obtained for and stored at $-20^{\circ} \mathrm{C}$ before processing for FC quantification and microbiological analysis.

\section{Laboratory methods}

Fecal calprotectin

Faecal samples were transferred on ice before FC extraction and ELISA analysis (Calpro AS, Lysaker, Norway) according to the manufacturer's instructions using duplicate appropriately diluted samples. FC concentration was determined relative to standard curves and expressed as $\mu \mathrm{g} / \mathrm{g}$ of faeces.

\section{Gut microbiota}

Gut microbiota that comprise the reported dysbiosis of CD were analysed using quantitative PCR (qPCR). Faecal DNA extraction was carried out using the FastDNA spin kit for soil and the FastPrep-24 bead homogeniser (MP Biomedicals, Solon, Ohio, USA) following the manufacturer's instructions. A SYBR Green qPCR analysis of faecal DNA was performed as described previously ${ }^{23}$ with the following modifications: amplified bacterial 16S rRNA genes served as standard templates as previously described except for Ruminococcus bromii L2-63 ${ }^{24}$ for the Ruminococcus primers; for E coli primers, E coli XL1-Blue was used. PCR reactions were performed in quadruplicate using a 7900HT Fast Real-Time PCR machine (Life Technologies). Data were analysed with SDS V.2.4 software (Life Technologies). Faecal water content was measured by lyophilisation and concentrations of bacteria expressed as $\log _{10}$ copies/gram dry faeces. Normalised proportions of bacteria were obtained by dividing specific group quantities through universal quantities. For details of primers and PCR conditions see online supplementary table S2.

\section{Peripheral blood T cell flow cytometry}

Whole blood, collected in lithium-heparin Vacutainer tubes (BD Bioscience), was stored at room temperature for $\leq 4 \mathrm{~h}$ before labelling with fluorescently conjugated monoclonal antibodies to detect CD3 T cells, naïve (CD45RA $\left.{ }^{+}\right)$and memory (CD45RA $\left.{ }^{-}\right)$ subsets of CD 4 and CD 8 T cells. Integrin $\alpha 4 \beta 7$ expression was assessed by labelling with anti- $\beta 7$ (see online supplementary methods for antibodies used).

Data were acquired using a LSRII 4-colour flow cytometer (BD Bioscience) and collected using fluorescence-activated cell sorting Diva software V.4.1.2 (BD Bioscience) using Flow-Count fluorospheres (Beckman Coulter) for absolute quantitation. Colour compensation was performed offline using Winlist V.6.0 (Verity Software House). 


\section{Genotyping}

Human DNA was extracted from whole blood using the phenol chloroform-isoamyl alcohol method. ${ }^{25}$ Genotyping was performed using the Illumina Infinium Immunochip ${ }^{26}$ which includes 70 of the $71 \mathrm{CD}$ disease risk loci described in Franke et al. ${ }^{19}$ To increase detection of NOD2 mutations and capture the enhanced risk of NOD2 compound heterozygosity, three NOD2 (rs2066845/ G908R, rs2066844/R702W and rs5743293/3020insC) were individually assessed. Cumulative genotype relative risk (GRR) for each participant was therefore calculated across a total of $72 \mathrm{CD}$ risk loci. A population distribution model of $\mathrm{CD}$ risk was generated using the REGENT R program ${ }^{27}$ and previously published ORs. ${ }^{19}$ Participants were categorised into reduced, average, elevated or high genotypical risk with reference to this model. ${ }^{28}$

\section{Intestinal permeability}

IP tests were carried out using lactulose-rhamnose tests. ${ }^{29}$ After overnight fasting, participants consumed $450 \mathrm{~mL}$ water containing $5 \mathrm{~g}$ lactulose, $2 \mathrm{~g}$ mannitol and $1 \mathrm{~g}$ L-rhamnose (BCM Specials, Nottingham, UK), followed by a $5 \mathrm{~h}$ urine collection. Aliquots from the pooled urine sample were stored at $-20^{\circ} \mathrm{C}$ until analysis.

Urinary sugar separation and detection was carried out by liquid chromatography-tandem mass spectrometry. Following microcentrifugation, supernatant was transferred to a LC-2000 platform autosampler (Jasco, Easton, Maryland, USA), and urinary sugars separated by high performance liquid chromatography using an amino column in hydrophilic interaction liquid chromatography mode. Sugars were quantified by electrospray tandem mass spectrometry, using the API 3200 (AB Sciex, Framingham, Massachusetts, USA).

\section{Power calculation}

The hypothesis was that siblings would demonstrate multiple aspects of a CD phenotype distinct from healthy controls. In order to calculate the number of participants required, we performed a power calculation based upon detecting lower concentrations of $F$ prausnitzii in siblings compared with healthy controls. This determined that 21 subjects would be required in each group to detect a difference in $F$ prausnitzii of $0.3 \log _{10}$ between siblings and healthy controls assuming a SD of 0.3 $\log _{10}$ (power 0.9 , significance level 0.05 ).

\section{Statistical analysis}

Normality was determined by visual assessment of histograms and Shapiro-Wilk test. Parametric analyses (one-way analysis of variance or Student's t test) and non-parametric analyses (Mann-Whitney or Kruskal-Wallis tests) with Tukey's or Bonferroni post hoc corrections applied as appropriate. Correlations between continuous variables were assessed with Pearson's correlation coefficients (PCCs). Associations between categorical variables were assessed using $\chi^{2}$ tests. Hierarchical multiple regression analysis determined dependence between variables, after controlling for the effect of other factors. The capacity of combined variables to discriminate between the three groups was determined using discriminant analysis (SPSS, version 18.0).

\section{RESULTS}

\section{Participants and genotype relative risk}

Twenty-two patients with CD in remission (mean CDAI 71 (SD 44.8)), 21 of their healthy siblings and 25 controls matched by group for age, gender, body mass index, ethnicity, smoking status and median number of siblings were recruited (see online supplementary table S3). Patients' disease characteristics were representative of northern European populations. All participants had normal CRP $(<5 \mathrm{mg} / \mathrm{L})$ at screening. Nine $(41 \%)$ patients and four (19\%) siblings carried at least one NOD2 mutation compared with four $(16 \%)$ controls $(p=0.054)$. No participant had evidence of either macroscopic or microscopic mucosal inflammation. Predictably, higher proportions of patients and siblings had an elevated or high GRR across the 72 CD risk loci assessed, compared with controls (table 1).

\section{Faecal calprotectin was increased in patients and siblings} FC was significantly higher in patients $(252 \mu \mathrm{g} / \mathrm{g}$, IQR $125-$ $775 \mu \mathrm{g} / \mathrm{g}$ ) compared with siblings $(36 \mu \mathrm{g} / \mathrm{g}$, IQR $17-65 \mu \mathrm{g} / \mathrm{g}$, $\mathrm{p}<0.003)$ and controls $(17 \mu \mathrm{g} / \mathrm{g}$, IQR 9-41 $\mu \mathrm{g} / \mathrm{g}, \mathrm{p}<0.003)$. The proportion with FC concentrations above normal $(50 \mu \mathrm{g} / \mathrm{g})$, was greater in patients $(21,95 \%)$ versus controls $(2,8 \%, \mathrm{p}<0.001)$, and siblings $(8,38 \%)$ versus controls, $(\mathrm{p}=0.028)$. FC was not affected by smoking status or disease location and did not correlate between related sibling-patient pairs ( $\mathrm{PCC}=-0.005, \mathrm{p}=0.983$ ).

\section{Intestinal permeability was increased in patients but not siblings}

Significantly more patients $(12,57 \%)$ had abnormal IP (ratio of urinary lactulose to rhamnose $>0.05$ ) than siblings or controls (6, (29\%) and $5(23 \%)$, respectively, $\mathrm{p}=0.044)$, but IP was not significantly different between siblings and controls. IP did not correlate between sibling pairs, (PCC $-0.024, p=0.917)$, but significantly correlated with FC across all groups (PCC 0.283 , $\mathrm{p}=0.023)$.

\section{A Crohn's-like dysbiosis, including reduced $F$ prausnitzii, was observed in siblings}

Compared with controls, patients had significantly lower concentrations of total bacteria, $F$ prausnitzii, Clostridia cluster IV, Ruminococcus spp., Roseburia spp., Bacteroides-Prevotella and Bifidobacterium adolescentis; a dysbiosis comparable with that previously described in $\mathrm{CD} .{ }^{3}$ Strikingly, siblings also had significantly lower concentrations of $F$ prausnitzii, Clostridia cluster

Table 1 The number of patients' siblings and controls observed in each of the four genotype relative risk (GRR) categories

\begin{tabular}{lcccc}
\hline GRR categories, $\mathbf{n}(\%)$ & Patients $(\mathbf{n}=\mathbf{2 2})$ & Siblings $(\mathbf{n}=\mathbf{2 1})$ & Controls $(\mathbf{n}=\mathbf{2 5})$ & $\mathbf{p}$ Values \\
\hline High (GRR $>4.185)$ & $3(14)$ & $1(5)$ & $0(0)$ & Pat-con 0.045 \\
Elevated (GRR 2.273-4.185) & $2(9)$ & $3(14)$ & $0(0)$ & Sib-con 0.046 \\
Average (GRR 0.445-2.277) & $11(50)$ & $12(57)$ & $11(44)$ & Pat-sib 0.726 \\
Reduced (GRR <0.445) & $6(27)$ & $5(24)$ & $14(56)$ & \\
\hline
\end{tabular}

Categorised GRR in patients with CD and siblings was significantly greater compared with controls. Genotyping was performed using the Illumina Infinium Immunochip as described in

$C D$, Crohn's disease. 
Table 2 Concentrations of bacterial groups and species in faecal samples were significantly different between patients, siblings and controls

\begin{tabular}{|c|c|c|c|c|c|}
\hline & \multicolumn{3}{|c|}{ Concentrations of bacteria, $\log _{10}$ copies/g median (IQR) } & \multirow[b]{2}{*}{ p Value } & \multirow[b]{2}{*}{ Between-group comparisons $p$ Values } \\
\hline & Patients $(n=22)$ & Siblings $(n=21)$ & Controls $(n=25)$ & & \\
\hline Faecalibacterium prausnitzii & $6.88(5.03-9.35)$ & $9.27(8.12-9.78)$ & $9.59(9.34-10.14)$ & $<0.001$ & $\begin{array}{l}\text { Pat-Sib: } 0.006 \\
\text { Pat-Con: }<0.003^{*} \\
\text { Sib-Con: } 0.048\end{array}$ \\
\hline Clostridia cluster IV & $7.76(6.54-9.56)$ & $9.34(8.76-9.93)$ & $9.69(9.48-10.26)$ & $<0.001$ & $\begin{array}{l}\text { Pat-Sib: } 0.045 \\
\text { Pat-Con: }<0.003 \\
\text { Sib-Con: } 0.030\end{array}$ \\
\hline Clostridia cluster XIVa & $9.86(9.07-10.31)$ & $10.13(9.64-10.46)$ & $10.19(9.85-10.85)$ & 0.099 & - \\
\hline Cluster IV Ruminococcus spp. & $7.05(5.94-8.29)$ & $8.75(8.04-9.33)$ & $9.55(8.37-10.02)$ & $<0.001$ & $\begin{array}{l}\text { Pat-Sib: }<0.003 \\
\text { Pat-Con: }<0.003 \\
\text { Sib-Con: } 0.084\end{array}$ \\
\hline Roseburia spp. & $9.19(7.35-9.85)$ & $9.34(7.21-9.70)$ & $9.92(9.39-10.15)$ & 0.004 & $\begin{array}{l}\text { Pat-Sib: } 1.000 \\
\text { Pat-Con: } 0.027 \\
\text { Sib-Con: } 0.009\end{array}$ \\
\hline Bacteroides-Prevotella & $8.83(8.35-10.05)$ & $10.16(9.50-10.70)$ & $10.48(10.08-10.80)$ & $<0.001$ & $\begin{array}{l}\text { Pat-Sib: } 0.009 \\
\text { Pat-Con: }<0.003 \\
\text { Sib-Con: } 0.639\end{array}$ \\
\hline Escherichia coli & $7.95(6.61-9.00)$ & $7.15(6.95-8.07)$ & $7.32(6.79-8.00)$ & 0.553 & - \\
\hline Bifidobacterium & $9.36(8.24-10.00)$ & $9.84(9.41-10.05)$ & $9.98(9.32-10.16)$ & 0.154 & - \\
\hline Bifidobacterium longum & $8.91(6.80-9.50)$ & $9.13(8.67-9.56)$ & $9.24(8.18-9.61)$ & 0.640 & - \\
\hline Bifidobacterium adolescentis & $5.76(4.69-8.78)$ & $8.87(5.50-9.30)$ & $9.20(7.88-9.56)$ & 0.018 & $\begin{array}{l}\text { Pat-Sib: } 0.228 \\
\text { Pat-Con: } 0.021 \\
\text { Sib-Con: } 0.714\end{array}$ \\
\hline Universal & $10.68(10.16-10.89)$ & $10.84(10.55-11.03)$ & $10.97(10.76-11.29)$ & 0.010 & $\begin{array}{l}\text { Pat-Sib: } 0.759 \\
\text { Pat-Con: } 0.015 \\
\text { Sib-Con: } 0.117\end{array}$ \\
\hline
\end{tabular}

The CD dysbiosis broadly reflected that previously described: reduced Firmicutes (including $F$ prausnitzii and Clostridia cluster IV), Bacteroides-Prevotella, $B$ adolescentis and total bacteria. Similar reductions in Firmicutes species including $F$ prausnitzii and Clostridia cluster IV also characterised the sibling dysbiosis, whereas concentrations of

Bacteroides-Prevotella, $B$ adolescentis and the overall concentration of bacteria were similar between siblings and controls. Bacterial DNA was extracted from faecal samples and detected using quantitative PCR probes as detailed in the methods and online supplementary methods.

*Due to Bonferroni correction of $p$ values, a minimum value of $<0.003$ is reported rather than $<0.001$.

$\mathrm{CD}$, Crohn's disease.

IV and Roseburia spp. compared with controls. However, concentrations of total bacteria, Bacteroides-Prevotella and $B$ adolescentis were not different between siblings and controls (table 2). The microbiota was also analysed as relative proportions of each group. A similar dysbiosis was described in the patients (see online supplementary table S4), with an additional finding that proportions of $E$ coli were increased in patients $(0.12 \%$ SD $3.95 \%)$ compared with controls $(0.02 \%$ SD $0.10 \%, \mathrm{p}=0.027)$ but were similar between siblings $(0.05 \%$ SD $0.14 \%)$ and controls $(\mathrm{p}=0.825)$.

Proportions of $F$ prausnitzii were significantly correlated between sibling pairs (PCC 0.436, $\mathrm{p}=0.043$ ) as were Clostridia cluster IV (PCC 0.532, $\mathrm{p}=0.011)$ and concentrations of Clostridia cluster XIVa (PCC 0.543, p=0.009). Smoking and immunosuppressant use were not associated with dysbiosis. Likewise, IP and FC were not independently associated with dysbiosis (data not shown). In addition to the effect of group in predicting the concentration of Bacteroides-Prevotella, $\left(\mathrm{R}^{2}=0.276, F_{1,66}=25.221\right.$, $\mathrm{p}<0.001$ ), there was a significant association between elevated/ high GRR and concentrations of Bacteroides-Prevotella $\left(\mathrm{R}^{2}=0.327, \mathrm{R}^{2}\right.$ change $\left.=0.050, F_{1,66}=4.838, \mathrm{p}=0.031\right)$, with grouping accounting for $28 \%$ and GRR for $5 \%$ of the variation. Concentrations and proportions of other bacteria did not vary according to GRR.

\section{Memory $T$ cells were increased in patients and their siblings}

The proportion of blood $\mathrm{T}$ cells that were CD45RA ${ }^{-}$memory $T$ cells was significantly higher in patients and siblings compared with controls, figure 1A. The higher proportion of memory T cells observed in patients and siblings compared with controls was significant in the CD4 T cell subset $(65 \%$, SD $13 \%$; 69\%, SD $12 \% ; 55 \%$, SD 13\%, respectively; $\mathrm{p}=0.001$ ), and a trend towards higher proportions of memory cells was also seen in CD8 T cells (53\%, SD 19\%; 57\%, SD 17\%; 45\%, SD 15\%, respectively; $\mathrm{p}=0.054)$. Total frequencies of peripheral CD3 T cells were significantly lower in patients compared with siblings or controls, online supplementary figure S1. This observation complements previous data indicating that blood $\mathrm{T}$ cell numbers are reduced in active CD. ${ }^{30}$ Absolute quantitation of $\mathrm{T}$ cell subsets revealed that the higher proportion of memory $\mathrm{T}$ cells was attributable to lower concentrations of CD45RA naïve T cells in patients and siblings compared with controls, whereas memory $\mathrm{T}$ cell frequencies did not differ significantly compared with controls, figure 1B-E.

\section{Naïve CD4 T cells exhibit enhanced gut-homing potential in patients and siblings}

The relative proportion of $\beta 7^{+}$and $\beta 7^{-}$cells in each $\mathrm{T}$ cell subset was analysed individually: proportions of CD4 naïve $\mathrm{CD}^{2} \mathrm{RA}^{+} \mathrm{T}$ cells expressing $\beta 7$ was significantly higher in patients and siblings compared with controls, figure 2A-D. Proportions of $\mathrm{T}$ cells expressing $\beta 7$ did not significantly differ between groups for any of the other $\mathrm{T}$ cell subsets. The proportion of memory $\mathrm{T}$ cells expressing $\beta 7$ (PCC 0.564, $\mathrm{p}=0.006$ ), but not the proportion of $\beta 7^{+}$CD4 naïve T cells, was significantly correlated between patient-sibling pairs.

\section{Correlation between factors in healthy siblings}

As demonstrated in the whole cohort, there was a negative correlation between the concentration of Bacteroides-Prevotella and GRR in siblings $(\mathrm{PCC}=-0.505, \mathrm{p}=0.019)$. Thus siblings with the 

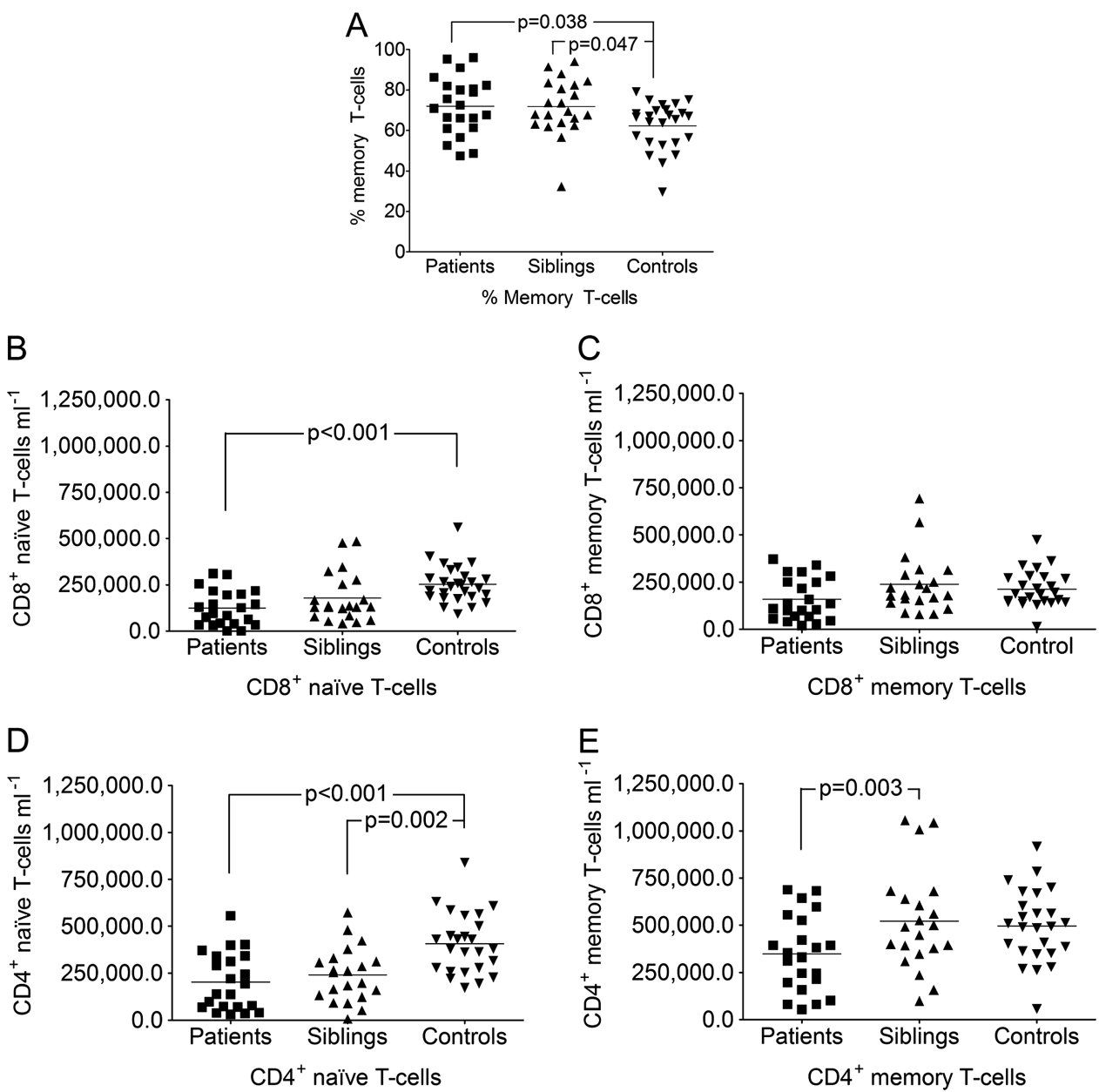

Figure 1 (A) Proportions of peripheral blood T cells with a memory (CD45RA-) phenotype were significantly greater in patients ( $n=22)$ and siblings $(n=21)$ compared with healthy controls $(n=25)$. (B-E) The predominance of the memory phenotype was attributable to a relative lack of naïve T cells. Although concentrations of T cells in patients were contributed to by the use of immunosuppressants by half of patients, concentrations of naïve $T$ cells were also reduced in immunosuppressant naïve siblings, and in the CD4 naïve T cell population this difference was significant. Whole blood was labelled with fluorescently conjugated monoclonal antibodies before acquisition of data using a LSRII flow cytometer.

highest genetic risk of CD shared aspects of the CD associated dysbiosis with their affected relative. In addition, there was a negative correlation between the concentration of Clostridia cluster IV and IP (PCC $=-0.514, p=0.017)$, the concentration of blood CD4 naïve $\mathrm{T}$ cells $(\mathrm{PCC}=-0.521, \mathrm{p}=0.016)$ and the proportion of CD4 naïve $\mathrm{T}$ cells which expressed $\beta 7$ integrin $(\mathrm{PCC}=-0.475$, $\mathrm{p}=0.030$ ). However, there was no correlation between Clostridia cluster IV and FC. Thus, reduced concentrations of Clostridia cluster IV in siblings were associated with several phenotypical characteristics that are associated with CD. Interestingly these correlations were not present in patients with established disease or healthy controls, which would support a role for this aspect of the dysbiosis in the pathogenesis of disease.

The multidimensional phenotype discriminates between patients, siblings and healthy controls

We next performed a discriminant analysis to determine whether combining genetic risk, FC, IP, gut microbiota and immunological parameters could distinguish between patients, 'at-risk' siblings and healthy controls, figure 3 . Independent variables were entered into the model and two discriminant functions (linear combinations of the variables, whose values are as close as possible within groups and as far apart as possible between groups) were calculated, with a combined $\chi^{2}=80.409$, degrees of freedom $(\mathrm{df})=20, \mathrm{p}<0.001$. The two discriminant functions accounted for $87 \%$ and $13 \%$ of the between-group variance, respectively. Function 1 maximally separated patients from controls. Function 2 maximally separated siblings from controls, and after removal of function 1 , the discriminating power of function 2 remained significant: $\chi^{2}=15.569,(\mathrm{df}=9), \mathrm{p}=0.049$. The loading matrix of correlations between predictor variables and discriminant functions suggested that variables contributing significantly to function 1 (providing maximal discrimination between patients and controls) were predominantly microbiological: concentrations of F prausnitzii, Ruminococcus spp., Bacteroides-Prevotella and Clostridia cluster IV, in addition to FC, (table 3). In contrast, the variables contributing significantly to function 2 (providing maximal discrimination between siblings and controls) were $\mathrm{T}$ cell attributes including the proportion of CD4 T cells expressing $\beta 7$ integrin and the proportion of $\mathrm{T}$ cells with a memory cell phenotype, as well as concentrations of Roseburia spp.

\section{DISCUSSION}

This is the first study to provide detailed and simultaneous analysis of the faecal microbiology, IP, FC and blood T cell phenotype of a cohort of clinically and genetically well-characterised patients with quiescent CD. Uniquely, the equivalent detailed assessments were also performed in healthy siblings of the patients with $\mathrm{CD}$ and matched unrelated controls. We report 
Figure 2 (A-D) Significantly higher proportions of CD4 naïve T cells expressed $\beta 7$ integrin in patients $(n=22)$ and siblings $(n=21)$ compared with controls, $(n=25)$. Relative abundances of $\beta 7^{+}$cells did not differ between groups for the other $T$ cell subsets. Whole blood was labelled with fluorescently conjugated monoclonal antibodies before acquisition of data using a LSRII flow cytometer.
A
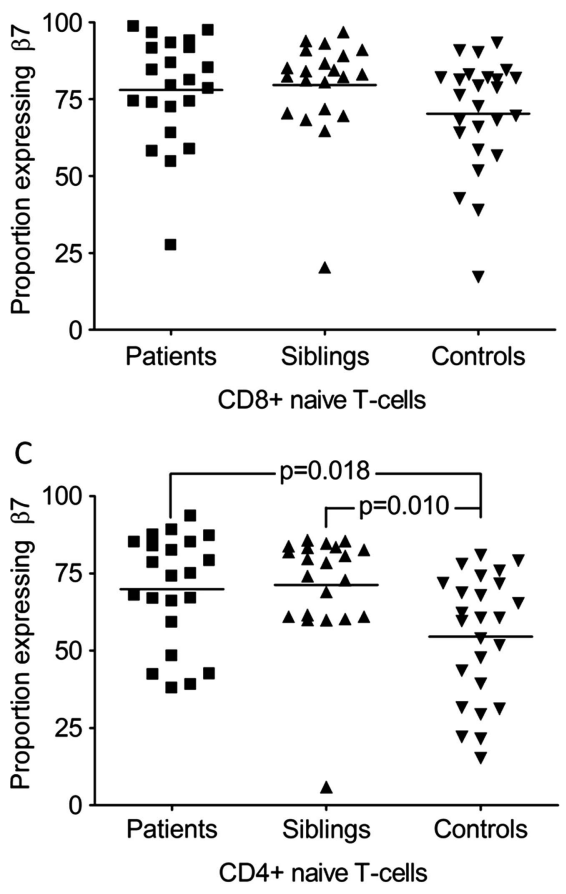

B

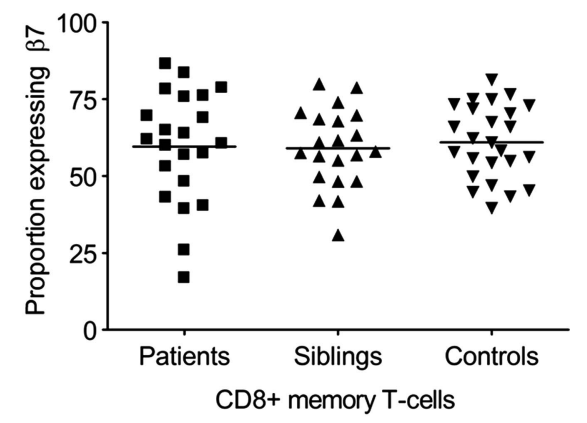

D

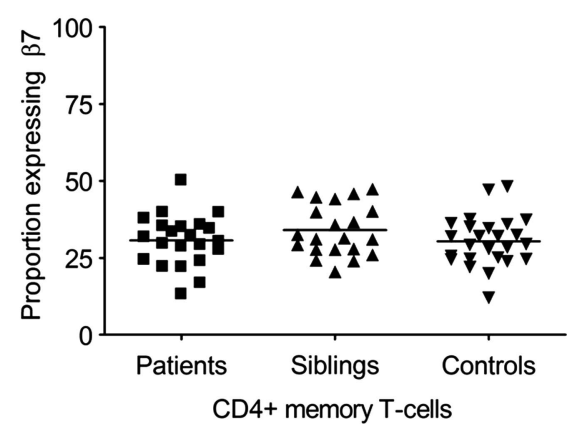

that siblings of patients with CD share multiple features of the CD phenotype in addition to genotype-related risk (table 4), including reduced Firmicutes such as $F$ prausnitzii, predominance of the memory phenotype in blood T cells, and elevated FC. We hypothesise that the unaffected sibling phenotype provides a unique window into factors important in CD pathogenesis. Studying healthy, 'at-risk' siblings allows the description of pathogenic factors in the absence of the influence of disease, surgery and pharmacotherapy that may distort the phenotype in patients. Finally, this study provides proof of principle that

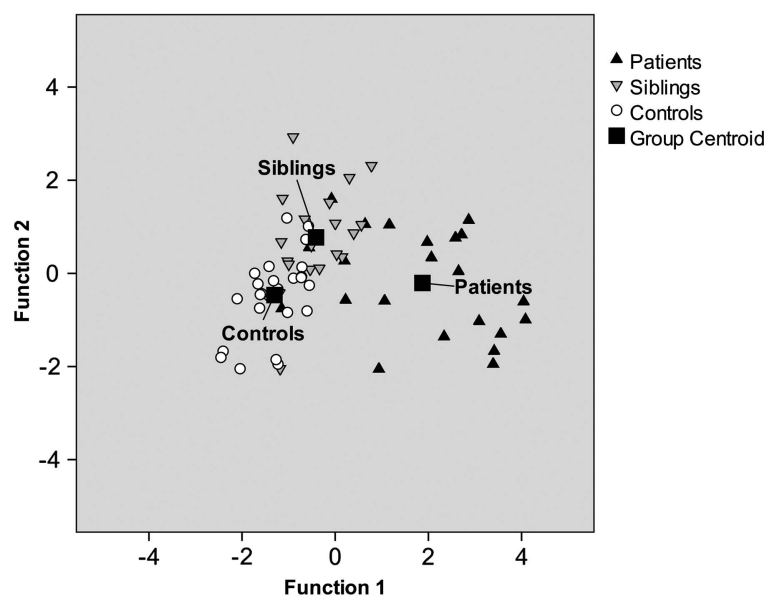

Figure 3 Direct discriminant function analysis revealed that the variables contributing significantly to function 1 (which provided maximal separation of patients with Crohn's disease from controls) were: elevated faecal calprotectin and altered faecal microbiota (reduced Faecalibacterium prausnitzii, cluster IV Ruminococcus spp., Bacteroides-Prevotella and Clostridia cluster IV). In contrast the variables contributing significantly to function 2 (which provided maximal discrimination of siblings from controls) were: increased $\beta 7$ integrin expression by circulating naïve CD4 T cells, an increased proportion of memory CD4 T cells and reduced faecal Roseburia spp. multidimensional phenotyping can be used to discriminate a population of healthy at-risk individuals from background risk controls.

\section{Genotype relative risk}

The patient and sibling cohorts were enriched for genetic loci associated with CD. The genetic validity of the cohort is

Table 3 Loading matrix of correlations between predictor variables and the two discriminant functions (figure 3 ) where function 1 provided most discrimination between patients and controls and function 2 provided maximal discrimination between siblings and controls

\begin{tabular}{|c|c|c|}
\hline & \multicolumn{2}{|c|}{$\begin{array}{l}\text { Pooled within-groups } \\
\text { correlations between } \\
\text { variables and discriminant } \\
\text { functions }\end{array}$} \\
\hline & Function 1 & Function 2 \\
\hline Faecalibacterium prausnitzii, $\left(\log _{10} / \mathrm{g}\right)$ & $-0.608^{*}$ & 0.050 \\
\hline Ruminococcus spp., $\left(\log _{10} / \mathrm{g}\right)$ & $-0.544^{*}$ & 0.043 \\
\hline Faecal calprotectin, $(\mu \mathrm{g} / \mathrm{g})$ & $0.528 *$ & -0.283 \\
\hline Bacteroides-Prevotella, $\left(\log _{10} / \mathrm{g}\right)$ & $-0.473^{*}$ & 0.037 \\
\hline Clostridia cluster IV, $\left(\log _{10} / g\right)$ & $-0.359^{*}$ & -0.163 \\
\hline Genotype relative risk & 0.251 & 0.043 \\
\hline $\begin{array}{l}\text { Proportion of } C D 4^{+} \text {naïve cells expressing } \beta 7 \\
\text { integrin, }(\%)\end{array}$ & 0.226 & $0.596 *$ \\
\hline Roseburia spp., $\left(\log _{10} / g\right)$ & -0.234 & $-0.466^{*}$ \\
\hline Proportion of memory T cells, (\%) & 0.196 & $0.439 *$ \\
\hline \multicolumn{3}{|c|}{$\begin{array}{l}\text { The contribution of each aspect of the multidimensional phenotype to each function } \\
\text { is reflected by the pooled within-groups correlations between variables and } \\
\text { discriminant functions. The values refer to the strength of the correlation of variables } \\
\text { with each discriminant function; the positive/negative symbol refers to the direction of } \\
\text { the correlation. } \\
\text { *Factors with a discriminant loading }>0.300 \text { are considered to contribute significantly } \\
\text { to the discriminant function. } \\
\text { CD, Crohn's disease. }\end{array}$} \\
\hline
\end{tabular}


Table 4 Summary of the phenotype of patients with $C D$ and their healthy siblings as compared with unrelated healthy controls

\begin{tabular}{|c|c|c|}
\hline & Patients with CD & Unaffected siblings \\
\hline Genotype relative risk & $\uparrow$ & $\uparrow$ \\
\hline Faecal calprotectin & $\uparrow \uparrow$ & $\uparrow$ \\
\hline Intestinal permeability & $\uparrow$ & $\leftrightarrow$ \\
\hline Gut microbiota & $\begin{array}{l}\downarrow \text { Faecalibacterium prausnitzii/Firmicutes* } \\
\downarrow \text { Bacteroides-Prevotella } \\
\downarrow \text { Bifidobacterium adolescentis } \\
\uparrow \text { Escherichia coli }\end{array}$ & $\downarrow \boldsymbol{F}$ prausnitzii/Firmicutes* \\
\hline $\begin{array}{l}\text { Blood } \\
\text { T cells }\end{array}$ & $\begin{array}{l}\uparrow \text { memory phenotype associated with naïve CD4 T cell } \\
\text { lymphopenia } \\
\uparrow \text { expression of } \beta 7 \text { integrin by naïve CD4 T cells }\end{array}$ & $\begin{array}{l}\uparrow \text { memory phenotype associated with naïve CD4 T cell } \\
\text { lymphopenia } \\
\uparrow \text { expression of } \beta 7 \text { integrin by naïve CD4 T cells }\end{array}$ \\
\hline
\end{tabular}

supported by higher genetic risk in siblings, a NOD2 mutation rate (patients $=41 \%$, controls $=16 \%$ ) similar to previous data ${ }^{31}$ and the ratio of siblings to patients carrying any NOD2 mutation. However, the amount of total disease variance that can be attributed to current genetic findings in $\mathrm{CD}$ is still low $(<15 \%),{ }^{1}$ hence genotyping alone is inadequate to accurately identify at-risk individuals ${ }^{12}$ and further research is required to identify features associated with predisposition towards CD. Combining such information with data on genotype may be a powerful method to detect risk.

\section{Fecal calprotectin and intestinal permeability}

Elevated FC and IP are associated with established CD, but only elevated FC appears to contribute to the sibling 'at-risk' phenotype in this study. ${ }^{13}$ This would suggest that breach of the intestinal barrier is not an early event in CD pathogenesis. Some data suggest that increased IP may only be apparent in relatives when exposed to triggers such as aspirin ingestion, ${ }^{15}$ and thus may not have been detected in the current study. The lack of correlation in FC between sibling pairs may imply that raised FC is not driven by genetic or early environmental factors shared between siblings.

\section{Dysbiosis}

Consistent with previous reports, ${ }^{3}$ the CD dysbiosis in our study included reduced $F$ prausnitzii, Clostridia cluster IV and Bacteroides-Prevotella. However, whether this dysbiosis predisposes towards $\mathrm{CD}$ or is an outcome of established disease cannot be determined by studying patients in isolation. Strikingly, siblings share elements of the CD dysbiosis, specifically depletion of the Firmicutes $F$ prausnitzii and Clostridia cluster IV, suggesting that these elements of CD dysbiosis are not purely consequent to established disease. The focus of the sibling dysbiosis on diminished Firmicutes populations is noteworthy given the range of mechanisms by which Firmicutes contribute to gut health ${ }^{32}$ and that phylum member $F$ prausnitzii predicts CD natural history. ${ }^{2}$ In contrast, the reduction in Bacteroides-Prevotella and $B$ adolescentis and increased $E$ coli seen in patients with CD were not found in their siblings, suggesting that these may become disturbed once disease is established. This study focused on aspects of the CD dysbiosis that have been reported previously and therefore used qPCR. Alternative methods such as $16 \mathrm{~S}$ pyrosequencing might detect unsuspected alterations in the microbiota in siblings that had not been reported as part of the dysbiosis in CD.
Few previous studies defined the gut microbiota of relatives of patients with $\mathrm{CD}$ with reference to healthy controls. One suggested that the organisms comprising the dysbiosis varied between patients with CD and their relatives. ${ }^{17}$ These findings are at odds with our study, where the sibling dysbiosis was similar to that in patients. This disparity may relate to previous studies including more heterogeneous groups of relatives. In contrast, the current study recruited only true siblings, potentially increasing the relevance of the dysbiosis we describe to early events in CD pathogenesis. Data from the current study suggest that loss of butyrate producers is common to patients with CD and their siblings, and is potentially the earliest microbiological step towards CD. Butyrate has recently been suggested to regulate the size of the colonic regulatory $\mathrm{T}$ cell pool and protect against murine colitis. ${ }^{33}$

In health, the intestinal microbiota is more similar between related than unrelated individuals. ${ }^{34}$ However, this similarity is reduced where one relative has $\mathrm{CD} .{ }^{35}$ Therefore, it would be predicted that siblings in the current study, discordant for disease phenotype, would have dissimilar microbiota. This was seen with Bacteroides-Prevotella, B adolescentis and E coli, but intersibling similarity was preserved for three bacterial groups within the Firmicutes phylum, suggesting this aspect of the CD dysbiosis is either genetically programmed, or that an early environmental exposure has a persistent impact on the microbiota, regardless of the later development of CD. The lack of association between GRR and Firmicutes abundance in the current study may favour prenatal, childhood or adolescent environmental factors in conferring this phenotype. Embryo transfer models demonstrate a maternal effect to determine the microbiota independent of genetic influence. ${ }^{36}$ Candidate maternally derived environmental factors include delivery by caesarean section and a protective effect of breastfeeding. ${ }^{37}$ However, a genetic influence over a CD-specific aspect of the dysbiosis was suggested by the association between GRR and Bacteroides-Prevotella. It may be speculated that the development of CD may require multiple microbiological 'hits', including environmentally acquired dysbiosis (to which siblings are also exposed), as well as genetically driven dysbiosis (more prominent in patients in this study).

\section{T cell phenotype}

We demonstrate that memory $\mathrm{T}$ cell phenotype predominance, previously described in active $\mathrm{CD}^{5}$ is also present in inactive $\mathrm{CD}$, particularly among CD4 T cells. However, in contrast to the redistribution of $\mathrm{T}$ cells from naïve to memory 
compartments previously reported in active $\mathrm{CD},{ }^{5}$ we show that in inactive CD blood naïve T cell frequencies are depleted in isolation. In patients with $\mathrm{CD}$, depletion of blood naïve CD4 T cells may therefore be a marker of disease that is independent of inflammation and may precede CD onset. Strikingly, a naïve CD4 $\mathrm{T}$ cell lymphopenia was demonstrated for the first time in unaffected siblings of patients with CD. It is unknown whether this naïve $\mathrm{T}$ cell lymphopenia reflects reduced generation of naïve $T$ cells, their sequestration outside the circulation or redistribution towards a memory population which has limited persistence in the circulation. It is tempting to speculate that peripheral homoeostasis of naïve T cells, which is driven by lowaffinity $\mathrm{T}$ cell receptor stimulation and interleukin 7 signalling (and may depend on the presence of the intestinal microbiota $^{38}$ ), may be disrupted in patients with $C D$ and their siblings. It is noteworthy that depletion of naïve CD4 T cells was associated with increased expression of $\beta 7$ integrin, a marker of gut tropism. In lymphocyte-replete animals, expression of the integrin $\alpha 4 \beta 7$ on naïve $T$ cells facilitates their entry into gut-associated lymphoid tissue. ${ }^{39} 40$ However, under lymphopenic conditions, IL-7 is induced which enhances $\alpha 4 \beta 7$ expression by naïve $\mathrm{T}$ cells and results in their migration into the intestinal mucosa. ${ }^{41}$ Therefore, perturbed homoeostasis of naïve CD4 T cells may be indicative of altered trafficking through organised lymphoid tissue and/or the intestinal mucosa in patients with $\mathrm{CD}$ and those at risk of $\mathrm{CD}$.

\section{Interactions between dimensions of the CD phenotype}

A unique feature of the current study is the simultaneous measurement of a variety of dimensions of the CD phenotype that permits interrogation of relationships between factors within and between groups. Thus the dysbiosis in siblings was associated with phenotypical features associated with CD (increased permeability, the concentration of blood CD4 naïve T cells and the proportion of CD4 naïve $\mathrm{T}$ cells which expressed $\beta 7$ integrin). Whereas, dysbiosis did not correlate with FC in either patients or siblings, indicating that it occurs independently of intestinal inflammation. The proportion of memory $\mathrm{T}$ cells correlated between patient-sibling pairs, but the lack of association between GRR and the proportion of memory T cells (and other features) may imply limited genetic determination. Consonant with this, a recent reanalysis of an early twin cohort estimated CD concordance in monozygotic twins to be $27 \%$ rather than the previously reported $58 \% .{ }^{42}{ }^{43}$ However, the contribution of genotype to CD pathogenesis will be better described as technological advances facilitate the measurement of 'non-code' genetic effects such as epistatic, epigenetic or parent-of-origin effects.

Finally, in addition to detecting correlations between individual factors, our study has allowed a multidimensional comparison of the $\mathrm{CD}$, sibling and healthy control phenotypes, enabling the identification of factors that discriminate healthy siblings of patients with CD from healthy controls. Microbiological factors such as $F$ prausnitzii and Bacteroides-Prevotella contributed more to discrimination of patients from healthy controls but were less significant in discrimination of the sibling phenotype, which was more dependent on immunological factors. Whether this sibling immune-characterised phenotype precedes CD onset or is an alternative phenotype of individuals who do not possess sufficient cumulative microbial or other triggers required to develop CD, may only be established with longitudinal studies.

Future studies investigating CD risk should simultaneously assess multiple dimensions. The $\mathrm{CD}$ and at-risk phenotypes described herein must be confirmed in other populations. In view of the breadth of measures reported in this study, we cannot exclude a type II error in some circumstances. In addition, newly uncovered features of the at-risk phenotype reported in this study such as the importance of $F$ prausnitzii, naïve CD4 $\mathrm{T}$ cell lymphopenia and altered expression of gut-homing markers provide fresh avenues of investigation into $\mathrm{CD}$ risk and pathogenesis. For example, the mechanisms of CD4 T cell lymphopenia in CD and 'at-risk' siblings remain to be determined, and the significance of potential pathogenic mechanisms such as Firmicutes short-chain fatty acid production, must be tested. Longitudinal studies are required to determine the capacity of the multidimensional analysis outlined in this study to predict disease in at-risk groups. Such longitudinal studies will be challenging, although it may be possible to perform repeated measurements at intervals in the offspring of patients with CD. This would have significant potential benefits to patients and their families as well as building on the insights into disease pathogenesis provided in this study.

Contributors CRH: study concept and design; recruitment of participants and acquisition of data; analysis and interpretation of data; drafting of the manuscript; critical revision of the manuscript for important intellectual content; statistical analysis; obtained funding. NEM: assistance with analysis and interpretation of immunology data; critical revision of the manuscript for important intellectual content. PL and FMF: assistance with analysis and interpretation of microbiology data; critical revision of the manuscript for important intellectual content; technical support. SM: assistance with recruitment of participants; critical revision of the manuscript for important intellectual content. KT: Human DNA extraction; assistance with analysis and interpretation of genetics data; critical revision of the manuscript for important intellectual content. NJP: assistance with analysis and interpretation of genetics data; critical revision of the manuscript for important intellectual content. TM: statistical advice, interpretation of statistical findings; critical revision of the manuscript for important intellectual content. AJS, KW and JOL: study concept and design; analysis and interpretation of data; critical revision of the manuscript for important intellectual content; obtained funding, study supervision.

Funding This research was funded by the charity Core.

Competing interests $\mathrm{CRH}$ was supported by a clinical research fellowship granted by the charity Core. FMF and PL received financial support from the Scottish Government Rural and Environmental Sciences and Analytical Services. NJP and KT were supported by the National Institute for Health Research (NIHR) Biomedical Research Centre based at Guy's and St Thomas' NHS Foundation Trust and King's College London.

Ethics approval Bromley Local Research Ethics Committee (reference 07/H0805/46).

Provenance and peer review Not commissioned; externally peer reviewed.

Data sharing statement Additional data are included in the online supplementary methods section for online publication.

\section{REFERENCES}

1 Jostins L, Ripke S, Weersma RK, et al. Host-microbe interactions have shaped the genetic architecture of inflammatory bowel disease. Nature 2012;491:119-24.

2 Sokol H, Pigneur B, Watterlot $\mathrm{L}$, et al. Faecalibacterium prausnitzii is an anti-inflammatory commensal bacterium identified by gut microbiota analysis of Crohn disease patients. Proc Natl Acad Sci USA 2008;105:16731-6.

3 Sartor RB. Microbial influences in inflammatory bowel diseases. Gastroenterology 2008:134:577-94.

4 Strober W, Fuss IJ. Proinflammatory cytokines in the pathogenesis of inflammatory bowel diseases. Gastroenterology 2011;140:1756-67.

5 Hart AL, Kamm MA, Knight SC, et al. Quantitative and functional characteristics of intestinal-homing memory T cells: analysis of Crohn's disease patients and healthy controls. Clin Exp Immunol 2004;135:137-45.

6 Garcia de TJ, Manzano L, Leal JC, et al. Active Crohn's disease patients show a distinctive expansion of circulating memory CD4+CD45RO+CD28null T cells. J Clin Immunol 2004;24:185-96.

7 Tibble JA, Sigthorsson G, Bridger S, et al. Surrogate markers of intestinal inflammation are predictive of relapse in patients with inflammatory bowel disease. Gastroenterology 2000;119:15-22.

8 Buhner S, Buning C, Genschel J, et al. Genetic basis for increased intestinal permeability in families with Crohn's disease: role of CARD15 3020insC mutation? Gut 2006;55:342-7.

9 Meuwis MA, Vernier-Massouille G, Grimaud JC, et al. Serum calprotectin as a biomarker for Crohn's disease. J Crohns Colitis 2013;7:e678-83. 
10 Calkins BM, Mendeloff Al. Epidemiology of inflammatory bowel disease. Epidemiol Rev 1986;8:60-91.

11 Hedin CR, Stagg AJ, Whelan K, et al. Family studies in Crohn's disease: new horizons in understanding disease pathogenesis, risk and prevention. Gut 2012;61:311-18.

12 Manolio TA, Collins FS, Cox NJ, et al. Finding the missing heritability of complex diseases. Nature 2009;461:747-53.

13 Thjodleifsson B, Sigthorsson G, Cariglia N, et al. Subclinical intestinal inflammation: an inherited abnormality in Crohn's disease relatives? Gastroenterology 2003;124:1728-37.

14 Munkholm P, Langholz E, Hollander D, et al. Intestinal permeability in patients with Crohn's disease and ulcerative colitis and their first degree relatives. Gut 1994;35:68-72.

15 Soderholm JD, Olaison G, Lindberg E, et al. Different intestinal permeability patterns in relatives and spouses of patients with Crohn's disease: an inherited defect in mucosal defence? Gut 1999;44:96-100.

16 May GR, Sutherland LR, Meddings JB. Is small intestinal permeability really increased in relatives of patients with Crohn's disease? Gastroenterology 1993:104:1627-32.

17 Joossens M, Huys G, Cnockaert M, et al. Dysbiosis of the faecal microbiota in patients with Crohn's disease and their unaffected relatives. Gut 2011;60:631-7.

18 Turpin W, Moreno-Hagelsieb G, Silverberg MS, et al. Specific Crohn's Disease (CD) associated risk alleles are associated with the composition of the intestinal microbiota in healthy First Degree Relatives (FDR) of CD subjects. Gastroenterology 2013; 142:S57

19 Franke A, McGovern DP, Barrett JC, et al. Genome-wide meta-analysis increases to 71 the number of confirmed Crohn's disease susceptibility loci. Nat Genet 2010;42:1118-25.

20 Kraus TA, Cheifetz A, Toy L, et al. Evidence for a genetic defect in oral tolerance induction in inflammatory bowel disease. Inflamm Bowel Dis 2006;12:82-8.

21 Joshi S, Lewis SJ, Creanor S, et al. Age-related faecal calprotectin, lactoferrin and tumour M2-PK concentrations in healthy volunteers. Ann Clin Biochem 2010;47:259-63.

22 Woodmansey EJ, McMurdo ME, Macfarlane GT, et al. Comparison of compositions and metabolic activities of fecal microbiotas in young adults and in antibiotic-treated and non-antibiotic-treated elderly subjects. Appl Environ Microbiol 2004;70:6113-22.

23 Ramirez-Farias C, Slezak K, Fuller Z, et al. Effect of inulin on the human gut microbiota: stimulation of Bifidobacterium adolescentis and Faecalibacterium prausnitzii. Br J Nutr 2009;101:541-50.

24 Dabek M, McCrae Sl, Stevens VJ, et al. Distribution of beta-glucosidase and beta-glucuronidase activity and of beta-glucuronidase gene gus in human colonic bacteria. FEMS Microbiol Ecol 2008:66:487-95.

25 Chomczynski P, Sacchi N. Single-step method of RNA isolation by acid guanidinium thiocyanate-phenol-chloroform extraction. Anal Biochem 1987:162:156-9.
26 Cortes A, Brown MA. Promise and pitfalls of the Immunochip. Arthritis Res Ther 2011;13:101

27 Crouch DJ, Goddard GH, Lewis CM. REGENT: a risk assessment and classification algorithm for genetic and environmental factors. Eur J Hum Genet 2013:21:109-11.

28 Goddard GH, Lewis CM. Risk categorization for complex disorders according to genotype relative risk and precision in parameter estimates. Genet Epidemiol 2010;34:624-32.

29 Mahmood A, Fitzgerald AJ, Marchbank T, et al. Zinc carnosine, a health food supplement that stabilises small bowel integrity and stimulates gut repair processes. Gut 2007:56:168-75.

30 Selby WS, Jewell DP. T lymphocyte subsets in inflammatory bowel disease: peripheral blood. Gut 1983;24:99-105.

31 Mathew CG, Lewis CM. Genetics of inflammatory bowel disease: progress and prospects. Hum Mol Genet 2004;13 Spec No 1:R161-8.

32 Flint $\mathrm{HJ}$, Scott KP, Louis $\mathrm{P}$, et al. The role of the gut microbiota in nutrition and health. Nat Rev Gastroenterol Hepatol 2012;9:577-89.

33 Smith PM, Howitt MR, Panikov N, et al. The microbial metabolites, short-chain fatty acids, regulate colonic Treg cell homeostasis. Science 2013;341:569-73.

34 Turnbaugh PJ, Hamady M, Yatsunenko T, et al. A core gut microbiome in obese and lean twins. Nature 2009:457:480-4.

35 Willing B, Dicksved J, Halfvarson J, et al. A pyrosequencing study in twins shows that GI microbial profiles vary with inflammatory bowel disease phenotypes. Gastroenterology 2010;139:1844-54.

36 Spor A, Koren O, Ley R. Unravelling the effects of the environment and host genotype on the gut microbiome. Nat Rev Microbiol 2011;9:279-90.

37 Malmborg P, Bahmanyar S, Grahnquist L, et al. Cesarean section and the risk of pediatric Crohn's disease. Inflamm Bowel Dis 2012;18:703-8.

38 Kieper WC, Troy A, Burghardt JT, et al. Recent immune status determines the source of antigens that drive homeostatic T cell expansion. J Immunol 2005;174:3158-63.

39 Bargatze RF, Jutila MA, Butcher EC. Distinct roles of L-selectin and integrins alpha 4 beta 7 and LFA-1 in lymphocyte homing to Peyer's patch-HEV in situ: the multistep model confirmed and refined. Immunity 1995;3:99-108.

40 Wagner N, Lohler J, Kunkel EJ, et al. Critical role for beta7 integrins in formation of the gut-associated lymphoid tissue. Nature 1996;382:366-70.

41 Cimbro R, Vassena L, Arthos J, et al. Interleukin-7 induces expression and activation of integrin alpha4beta7 promoting naive T-cell homing to the intestinal mucosa. Blood 2012;120:2610-19.

42 Halfvarson J. Genetics in twins with Crohn's disease: less pronounced than previously believed? Inflamm Bowel Dis 2011;17:6-12.

43 Tysk C, Lindberg E, Jarnerot $\mathrm{G}$, et al. Ulcerative colitis and Crohn's disease in an unselected population of monozygotic and dizygotic twins. A study of heritability and the influence of smoking. Gut 1988;29:990-6. 


\section{GUT}

\section{Altered intestinal microbiota and blood T cell phenotype are shared by patients with Crohn's disease and their unaffected siblings}

Charlotte R Hedin, Neil E McCarthy, Petra Louis, et al.

Gut 2014 63: 1578-1586 originally published online January 7, 2014 doi: 10.1136/gutjnl-2013-306226

Updated information and services can be found at:

http://gut.bmj.com/content/63/10/1578.full.html

\section{These include:}

Data Supplement

"Supplementary Data"

http://gut.bmj.com/content/suppl/2014/01/07/gutjnl-2013-306226.DC1.html

References This article cites 43 articles, 16 of which can be accessed free at: http://gut.bmj.com/content/63/10/1578.full.html\#ref-list-1

Email alerting service

Receive free email alerts when new articles cite this article. Sign up in the box at the top right corner of the online article.

Topic Articles on similar topics can be found in the following collections Collections

Notes

To request permissions go to:

http://group.bmj.com/group/rights-licensing/permissions

To order reprints go to:

http://journals.bmj.com/cgi/reprintform

To subscribe to BMJ go to:

http://group.bmj.com/subscribe/ 